\title{
Geometric Instruments for the Orientation and Measurement: The Astrolabes
}

\author{
Barbara Aterini \\ University of Florence, Florence, Italy \\ Email: barbara.aterini@unifi.it
}

How to cite this paper: Aterini, B. (2019). Geometric Instruments for the Orientation and Measurement: The Astrolabes. $A d$ vances in Historical Studies, 8, 58-78. https://doi.org/10.4236/ahs.2019.81004

Received: February 2, 2019

Accepted: March 16, 2019

Published: March 19, 2019

Copyright (C 2019 by author(s) and Scientific Research Publishing Inc. This work is licensed under the Creative Commons Attribution International License (CC BY 4.0).

http://creativecommons.org/licenses/by/4.0/

\begin{abstract}
The astrolabe, stereographic projection of the celestial sphere on the plane of the equator, simulates the apparent rotation of this sphere around the Earth, with respect to a certain latitude, and allows to establish the relative position of the stars at a given moment. To understand its meaning and use it is necessary to recall out the main elements of astronomical geography. From the III century BC onwards, various types of astrolabe were built. Among the tools used in the course of the centuries for orientation and measurement, it is the one that perfectly performed both functions, providing measurements related to different fields of investigation: astronomy, chronometry, orientation, measurements (length-height-depth), astrology.
\end{abstract}

\section{Keywords}

Astrolabe, Stereographic Projection, Measuring Instruments, Astronomy

\section{Introduction}

The tools used in the course of the centuries for orientation and measurement are varied, but what fulfilled both functions in a precise manner is certainly the astrolabe. The astrolabe is a flat representation of an armillary sphere. It simulates the apparent rotation of the celestial sphere around the Earth, is relative to a certain latitude, and allows to establish the relative position of the stars at a given moment. Leaving aside deliberately in this writing the detailed description of the instrument, for which we refer to the previous study on the topic (Aterini, 2019), we want to point out his functions based on fundamental geometric-projective principles, as well as clarifying the elements of astronomical geography that come into play for its operation. The astrolabe is a metallic instrument, a sort of hollow disk, called a mother, with a diameter of 10 to 40 centimeters, with the two faces (front and back) covered with inscriptions; inside it 
contains a series of disks (one for each latitude where you want to use it). A rather simple invoice object that however offers multiple possibilities of use and manages to solve the problems of astronomy, celestial mechanics, chronological events and trigonometry, and also, if necessary, the curves related to Kabbalah and astrology, and then become a computer and a real vademecum in which for centuries both the astronomer and the sailor have found the information currently supplied to them by the nautical ephemeris, the logarithm tables and the sextant. To understand the principles on which the astrolabe is based and which are the laws that regulate its use it is necessary to point out the main elements of astronomical geography. The Earth, planet of the solar system, is a celestial body studied as an ellipsoid of rotation, slightly flattened at the poles, and is called "geoid", but for the purposes of gnomonic it can be considered spherical. The position of any place on the surface of the Earth is identified by means of a pair of geographical coordinates; their reference system is anchored to the rotation axis of the Earth, which meets the earth's surface in the poles. The maximum circle (or circumference) perpendicular to the earth's axis is the equator, all the minor circle ones parallel to it are called "parallels". The maximum circles passing through the poles are called "meridians" and among them the one passing through Greenwich is the reference meridian 0 . The two coordinates referred to this system are the latitude and longitude that are measured in degrees, first and second. By definition: latitude $\varphi$ is the arc of the meridian that passes through the place, longitude $\lambda$ is the equator arc between the reference meridian 0 and the meridian of the place. The terrestrial ray passing through a given location and for the center of the sphere is called the "vertical" of the place. Given the minimum size of the Earth compared to its distance from the Sun, this can be considered a point, that is, concentrated in its center, and the solar rays that reach it can all be considered parallel to each other. The geographic coordinate system is objective, but since the observer who is in a given place on the earth's surface needs to refer to himself everything he sees, a second coordinate system, called "local", is needed. The center of this system is the position of the observer, whose vertical is projected upward in a $\mathrm{Z}$ direction called "zenith", while the diametrically opposite direction is called "nadir". The plan tangent to surface of Earth at the point where the observer is located is called the "horizon"; on this floor is the local meridian, which is oriented in the directions of the geographic poles and identifies the cardinal points North and South on the horizon. To indicate the direction of any object A we consider the vertical plane that contains $A$ and the vertical line for it. The direction of the objects is identified by means of two coordinates: azimuth and height. We can say that: the azimuth Az of an object is the angle on the horizon between the north direction of the local meridian and the trace of the vertical plane that contains the object; the height $\mathrm{h}$ of an object is the angle on its vertical between the horizon and the direction of the object. These two local coordinates, $\mathrm{Az}$ and $\mathrm{h}$, define only the direction of the object, not its position (Fantoni, 1988).

The Earth is subject to two movements: rotation on itself around the polar 
axis and rotation around the Sun, its fixed star (Figure 1). The first movement takes place counterclockwise (looking from the north) lasts 24 hours and determines the alternation of days and nights, separated by the phenomena of sunrise and sunset. The second movement, i.e. the rotation of the Earth around the Sun, it takes place in an anti-clockwise direction (looking from the north) on an elliptical orbit of which the Sun occupies one of the fires. The duration of a complete tour, called "tropic year", is 365.2422 days. The axis of rotation of the Earth is inclined $23^{\circ} 27^{\prime}$ with respect to the axis of the elliptical orbit around the Sun. This arrangement of the Earth's axis, in the first place, determines the alternation of the seasons, since the two hemispheres of the Earth receive less or more insolation depending on the elliptical orbit in which the planet is located in its annual tour. Furthermore, the Earth's axis points towards a specific star of the firmament, the Stella Polare, which thus materializes the direction of the North Pole.

The man who is on Earth sees the stars moving around himself according to "apparent" motions. We imagine that the observer is the center of a sphere of arbitrary radius; on this sphere the stars and their apparent motions are projected centrally, as the observer sees them. In other words, the visible cosmos is transferred to that apparent sphere that man calls "heavenly vault" and in cosmography it is called the "celestial sphere". The center of this sphere is the Earth, given its small dimensions; the geographic poles are projected on the celestial poles North (PN) and South (PS); meridians, parallels and equator look the same. The zenith of the observer in latitude $\varphi$ is in $\mathrm{Z}$ and its direction (vertical of the observer) is inclined on the equator as the latitude $\varphi$; $\mathrm{Z}^{\prime}$ is the Nadir; the horizon is projected in the maximum circle perpendicular to $\mathrm{ZZ}^{\prime}$; the cardinal points are arranged with the North on the extension of the ZPn arc. The observer can see all that is above the horizon, that is, everything in the hemisphere that has the zenith $\mathrm{Z}$ as the pole.

We transfer on the celestial sphere the apparent motions of the Sun, deriving from the real ones of the Earth (Figure 2 \& Figure 3): we start from the annual motion of the Earth on the elliptical orbit: if we stop the Earth the apparent motion of the Sun manifests itself on the celestial sphere as a maximum inclined

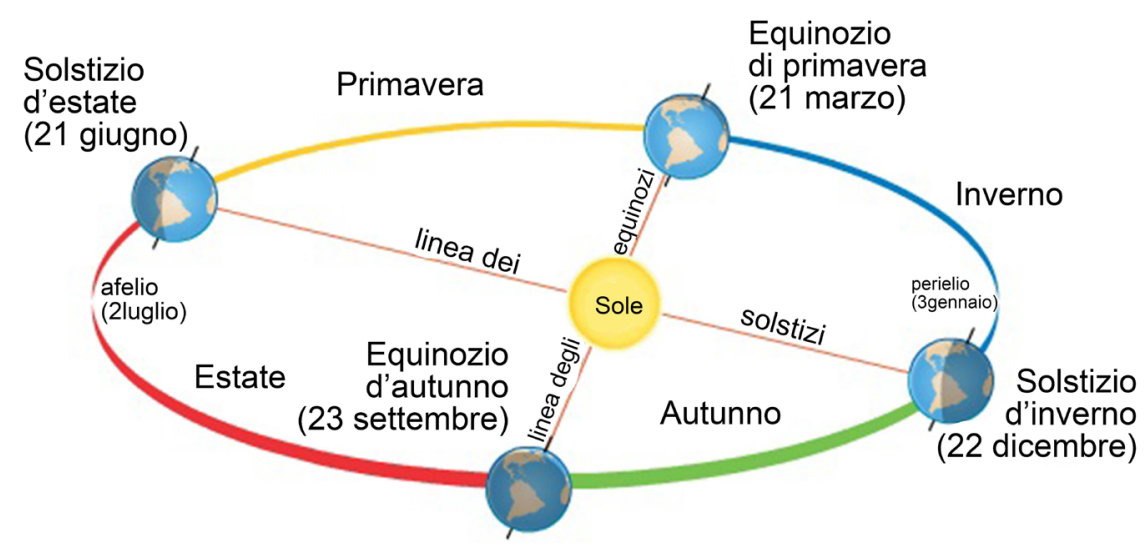

Figure 1. The movements of the Earth. 


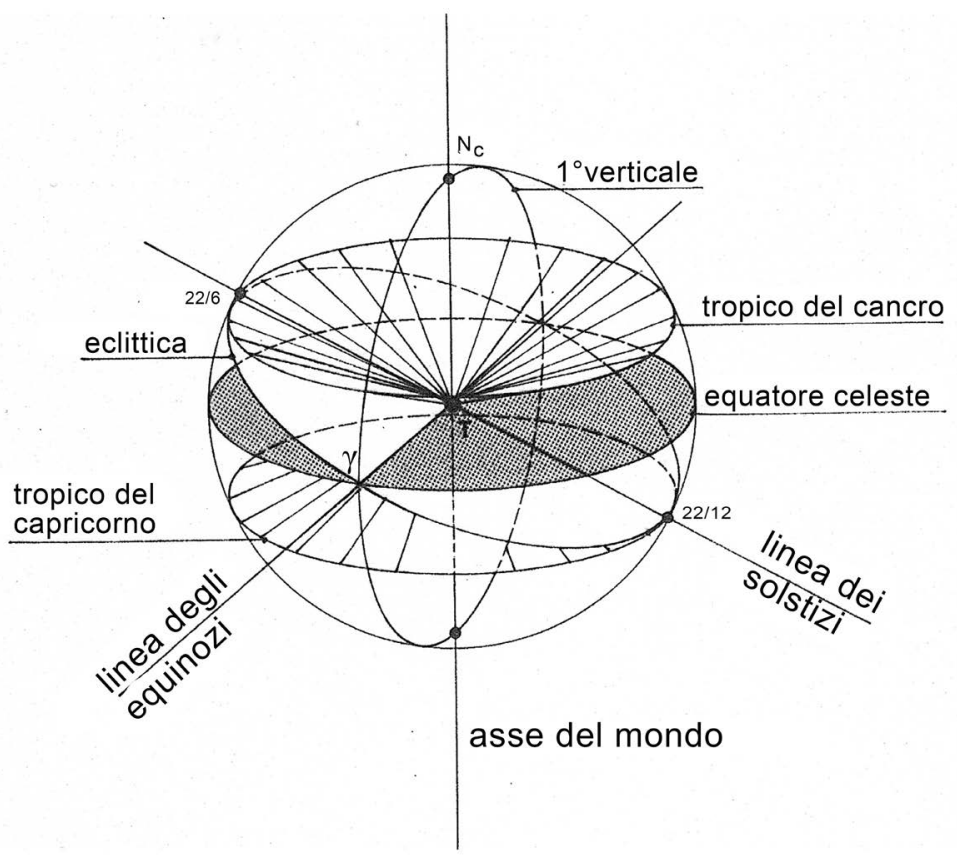

Figure 2. The celestial sphere.

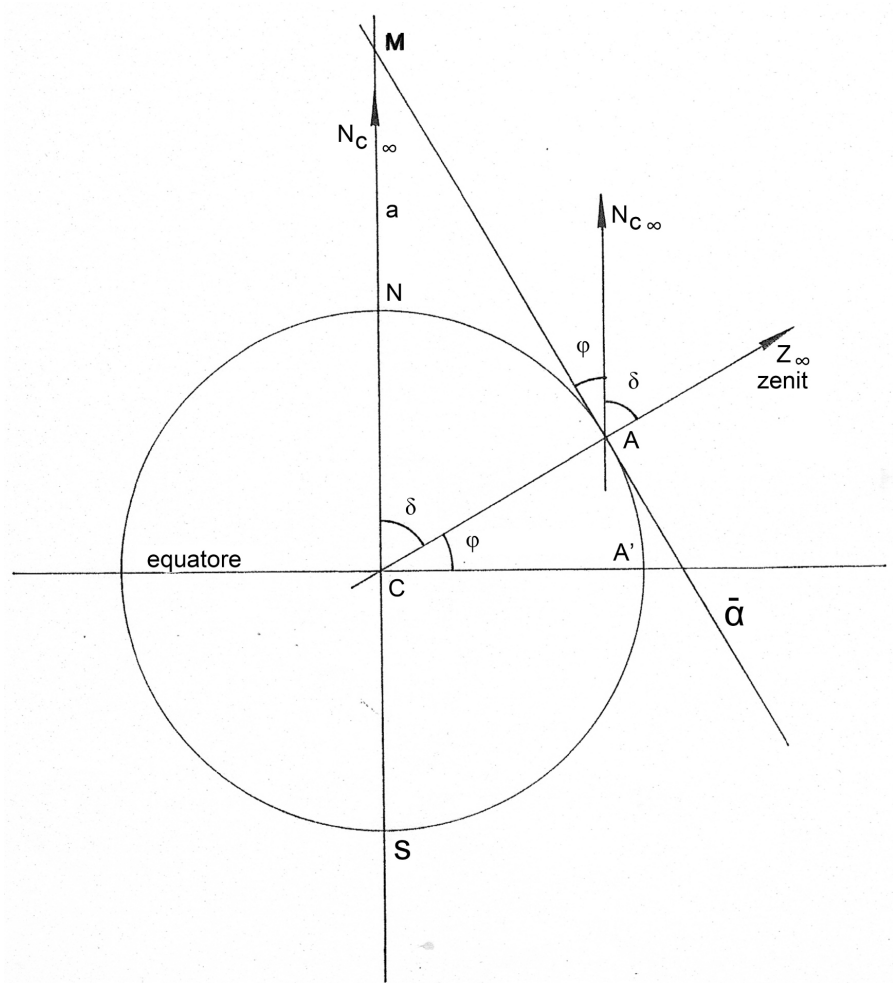

Figure 3. Section of the celestial sphere: on it the objective positions of the stars are identified by means of a system of celestial coordinates called "equatorial".

circle of $23^{\circ} 27^{\prime}$ on the level of the equator; on it the Sun moves counterclockwise completing a lap in 365.2422 days. This circle, which is called "ecliptic", meets the equator in two points: $\gamma$ and $\gamma$; the gamma point $\gamma$, known as the "vernacu- 
lar point', is where the Sun begins its journey in the northern hemisphere and is important both in cosmography and in the gnomonic (Figure 2). On the celestial sphere the objective positions of the stars are identified by means of a system of celestial coordinates called "equatorial" very similar to the geographical coordinates. The coordinate on the equator is called "straight ascension" and is counted in degrees from $0^{\circ}$ to $360^{\circ}$ or in hours from 0 to 24 in an anti-clockwise direction starting from the gamma point; the correspondence between degrees and hours is $15^{\circ}=1$ hour. The second equatorial coordinate is called declination $\delta$ (fundamental element in solar clocks) and is measured on the celestial meridian of the star (also called the "hour circle") starting from the equator from $0^{\circ}$ to $+90^{\circ}$ towards North and from $0^{\circ}$ to $-90^{\circ}$ towards the South. The variation of the solar declination in the course of a year increases from -23.5 to +23.5 while the Sun rises on the ecliptic from the winter solstice to the summer one, then it comes down from the summer solstice to the winter solstice. While the apparent circle of the Sun causes the succession of the seasons, the rotation of the Earth on itself transfers a series of phenomena on the celestial sphere, among which the alternation of day and night. The real motion of the Earth around the PnPs axis determines an apparent rotation of the whole celestial sphere and the stars. To follow this transit of the star in relation to the passage of time another local coordinate has been introduced: the time anglet, i.e. the angle between the lower meridian and the meridian containing the star, is measured in hours, from 0 to 24, clockwise. The celestial meridians, when referred to the time angle are called "hourly circles" and the half-planes that generate them are called "hourly half-planes". The diurnal rotation of the hourly half-planes, or of the Sun's hourly circle, defines the "hour". The evolution of the local coordinates of a star (azimuth, height, time angle) and all the connected elements (diurnal arch, night arc, hours, azimuth at sunrise and sunset, height of culmination, etc...) depend on the latitude of the observer and by declination of the star. The Sun undergoes the apparent daytime rotation due to the real rotation of the Earth but, we have seen, its declination oscillates between +23.5 and -23.5 (Figure 4 \& Figure 5).

This means that the Sun describes daily a circle of variable declination during the year and included between the two extreme circles EE' (summer solstice) and II' (winter solstice). Consequently, the height of the Sun above the horizon is variable, depending on the date, from a maximum (summer solstice) to a minimum (winter solstice). At the equinoxes, when its declination is $0^{\circ}$, its path is divided by the horizon into two equal parts: day and night are both 12 hours. The circular crown between the two extreme circles of the solar declination represents the area of the celestial sphere in which the Sun makes the annual path around the Earth. It takes the name of "zodiacal band" because the ancients divided the ecliptic into 12 sections of $30^{\circ}$ (three for each season) called with the twelve "zodiacal signs"; each sign, corresponding to a specific constellation, included the path of the Sun in a month and, in turn, was divided into $30^{\circ}$ each corresponding to about a day. To identify the various points of the zodiac on the ecliptic it may be useful to consider another coordinate: the "celestial longitude" 


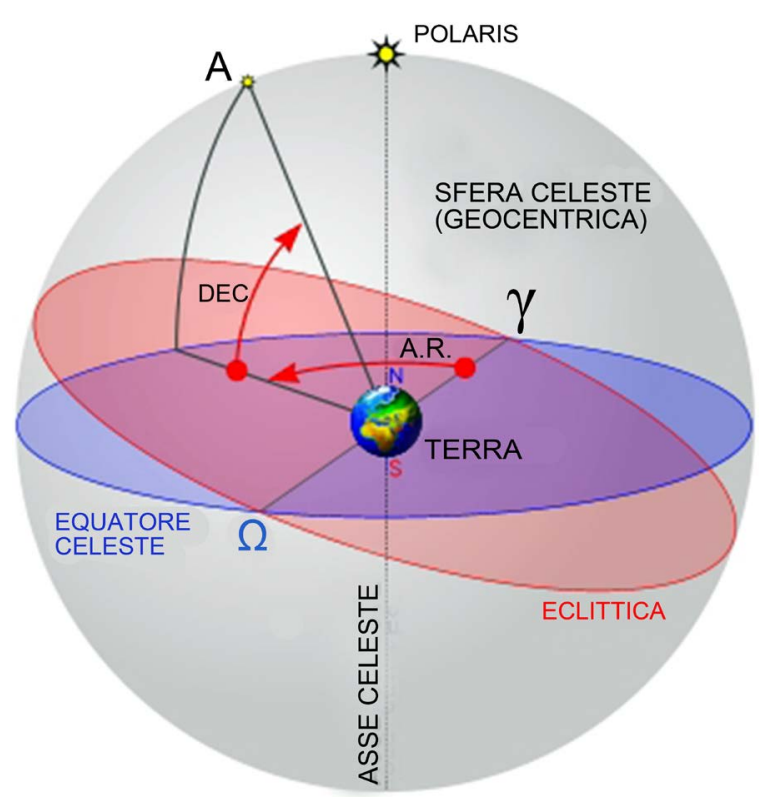

Figure 4. The celestial sphere.

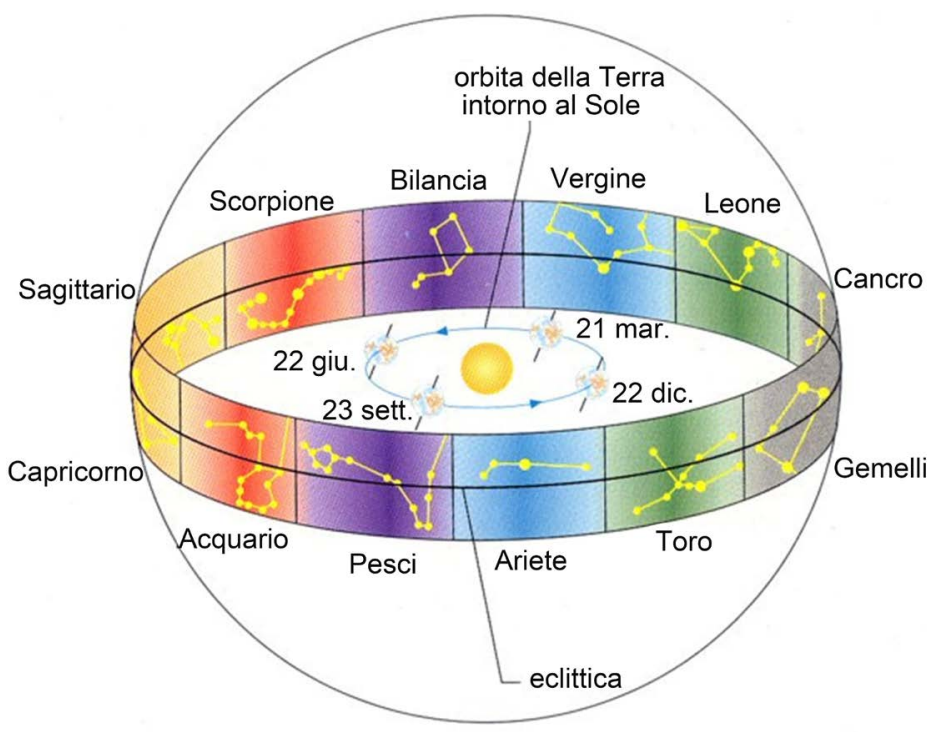

Figure 5. The zodiac band.

$\lambda$ which is measured on the ecliptic from $0^{\circ}$ to $360^{\circ}$ counterclockwise starting from the gamma point (Figure 6).

\section{The Astrolabe and Projective Geometry}

In the field of the gnomonic the best way to know the azimuth of the Sun is to plant a vertical stick on the ground and use the horizon as a reference plane: in this way the shadow of the stick referred to the South cardinal point marks directly on the horizon the value of the azimuth $\mathrm{Z}$. In the past two projections of this type have been used, both with the reference plane on the horizon: the stereographic projection with the projection center in one of the poles; with this 


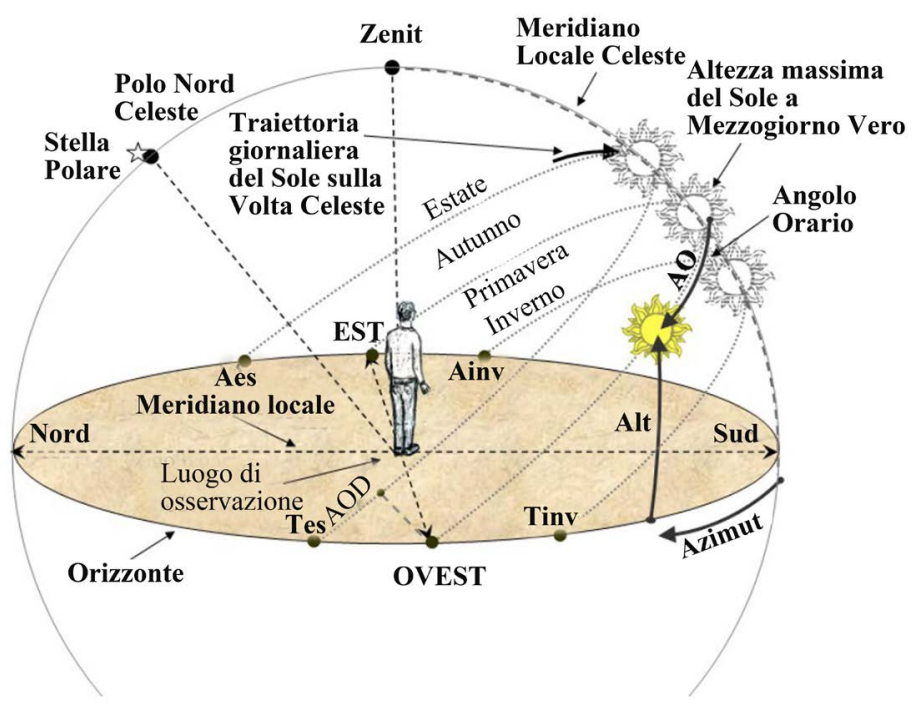

Figure 6. The observer is the center of the celestial sphere.

method the azimuth clock called "horizontal astrolabe" was obtained; this is a projective azimuthal solar clock, obtained by projecting stereographically on the horizon the lower hemisphere of the celestial sphere, with point of projection on the North Pole. Its name derives from the stereographic projection, it normally used for the construction of astrolabes which, however, generally used the equatorial plane as a reference plane; instead the orthographic projection places the point of projection to infinity on the vertical of the observer; with this method the azimuthal clock called "analemmatic" was obtained. The link between the solar clock and the astrolabe is evident even if the astrolabe is a more sophisticated instrument than the solar clock: it not only represents the whole celestial sphere, but allows you to follow the movements of the celestial bodies and therefore to solve all the problems related to these movements (Figure 7).

The astrolabe is a simple tool but composed of several overlapping elements (Aterini, 2019). The main part is the body of the astrolabe, a flat disc on the back, and concave on the front. In this cavity of the body, bounded by a circular crown or margin, there are places for up to nine discs, each built on a different latitude; obviously the disc of the place where you want to use the astrolabe is above the margin level. The margin is divided into four concentric parts that correspond to the measurement of decigrades, degrees, hours and the identification of winds according to their origin. Once the disk is fixed by a tooth, is placed above this the aracnea, a circular metal net that can rotate around the center and allows the disc to be seen. Above the aracnea is the ruler, a line with two long arms so as to reach the edge of the astrolabe, which rotates autonomously around the center of the astrolabe. Along the diameter, or line of trust, various information is read, obtained from the combination of aracnea with the latitude disk and the margin. The back of the instrument, presents sign of various kinds: a series of circular crowns, that is the zodiacal circle and the perpetual calendar, delimit the central part. The zodiacal circle is characterized by the 


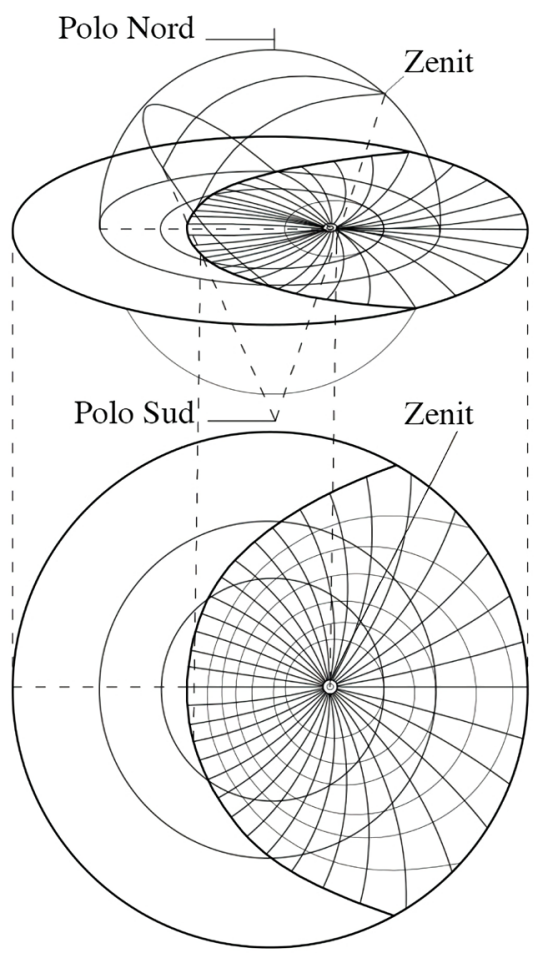

Figure 7. The Stereographic projection of the sphere is the operation by which the points of the surface of the sphere are projected from a fixed point $P$, called pole, on a plane $\pi$ parallel to the tangent plane at the sphere in the pole. In this way a double correspondence (homology) is achieved between the points of the sphere and those of the plane $\pi$ which preserves the form and is therefore said to conform. The angles are maintained, so the projection of a circle $\mu$ of the spherical surface from a pole $\mathrm{V}$ is still a circle $\mu$. In stereographic projections the point of view is located on the earth's surface and in opposite site to that of the plane's tangency. The distances between the parallels are not proportional to the real ones. In orthographic projections the point of view is located at infinity and the projection rays are parallel. The parallels are as close as possible the further you move away from the point of tangency.

measurement of the revolution angle in degrees and decigrades and by the division into twelve astrological signs, of thirty degrees each. Within the zodiacal circle we find a perpetual circular calendar arranged so that each day corresponds to a degree of the ecliptic. The calendar, however, does not have the same division of the outer circle, in fact the number of days of a year (365 and a quarter) established by the Chinese, 2400 years BC, does not allow the division in twelve months of exactly thirty days. Furthermore, due to the "precession of the equinoxes" the start dates of the seasons (equinoxes and solstices) do not divide the year into four equal periods. So to resolve this discrepancy the calendar is eccentric to the outer ring. The half-line that starts from the center of the zodiacal circle and passes through the center of the calendar is called "apse line" and touches the outer circumference at a point called the "solar Auge". In the astrolabe the auge is the point of contact of the two circles and currently is at about $12^{\circ} 50^{\prime}$ and is moving slowly to the left (equinoctial precession). So by measuring the "solar auge" it is possible to establish the era of manufacture of an 
astrolabe. The central circle, concentric with the astrolabe, is divided by the horizontal diameter into two graphical systems: the sundial and the "squadro a ombra".

The sundial is formed by circumference arches passing through three known points: from the center of the astrolabe and from the pair of points formed every $15^{\circ}$ on the upper hemicycle of the instrument (Figure 8). The set of arches of the sundial differs from that of the unequal hours due to its universality of latitude. The "squadro a ombra", located at the bottom of the central circle, is a diagram in function of the apparent diurnal path of the Sun. In fact it produces shadows that are proportional both to the height of the illuminated objects and to the position of the Sun itself. Appropriate lines directed towards the center of the astrolabe divide the graphical scheme into a number of parts relative to the measurement system used by the people who used the instrument. In ancient instruments the 12 is the most frequent number. Through the trigonometry applied to the angles of solar inclination and the length of the shadows produced by the gnomon, it is possible to know the height of objects only by measuring their shadows. In the astrolabe the inclination of the Sun is known through the use of the alidada, while the gnomon is represented by the line of the "squadro a ombra" intersected by the vertical diameter. The alidade is superimposed on the back of the astrolabe, a revolving rod that has wings perpendicular to the body plane, pierced so as to be able to look at a point-like body. This is the most significant part of the instrument and along the "line of trust", which extends as far as diameter, the divisions of the signs of the elliptical are traced. The central pivot is the connecting element of all the parts of the astrolabe; It is pierced in such a way as to accommodate the horse, a clasp that locks the instrument, but can easily be removed to change the latitude disks. Furthermore on the astrolabe is a jockstrap, that is a ring in which you can put a finger to hold the instrument while it is used (Figure 9).

\section{Types of Astrolabes}

Since ancient times, astronomers have used material models of the sky to make

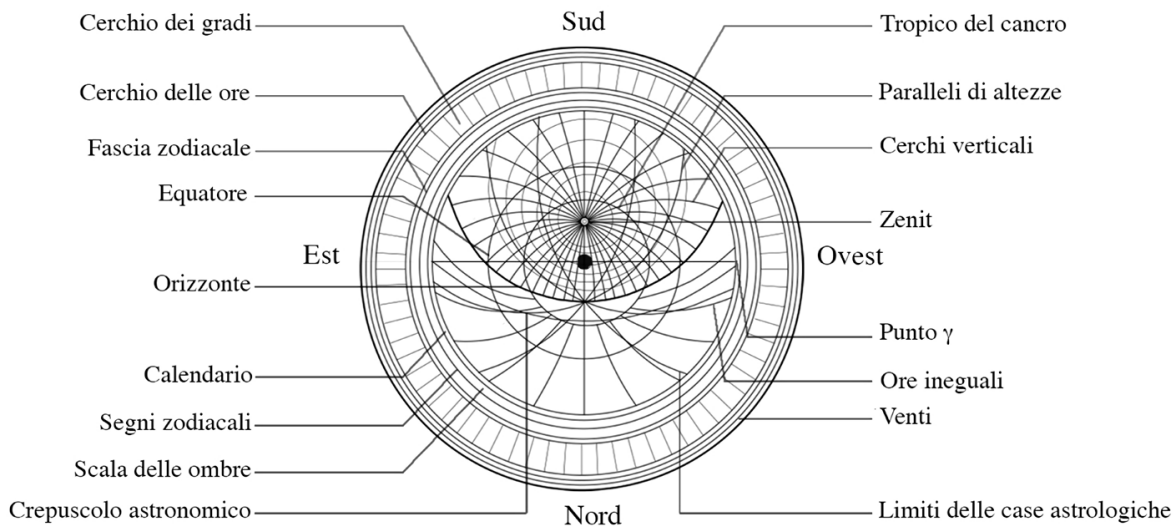

Figure 8. Reading of the astrolabe. 


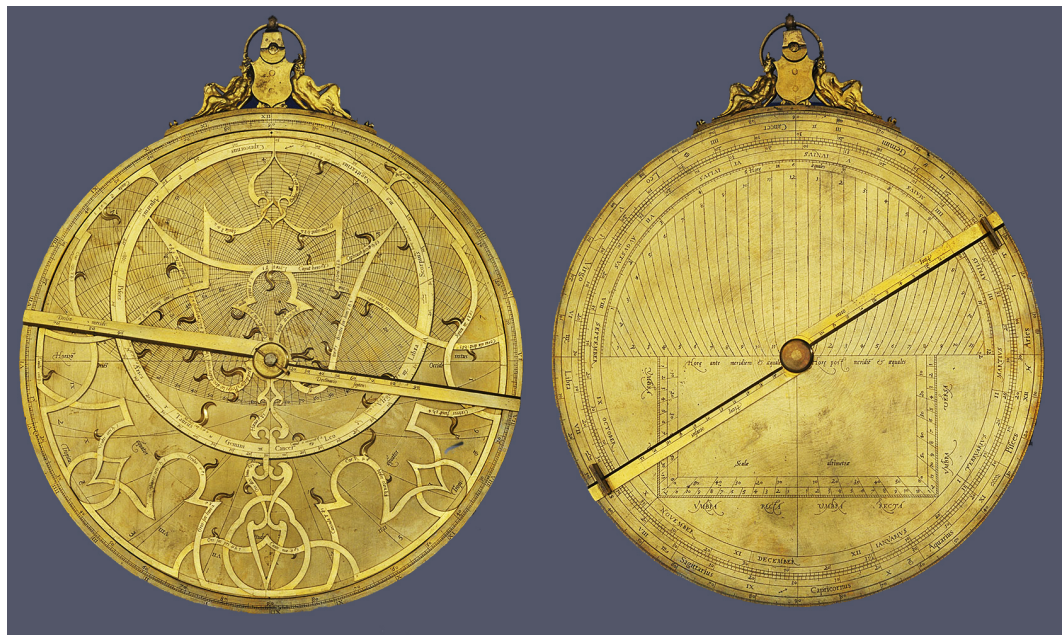

Figure 9. The two sides of the astrolabe: right the mother with the aracnea; on the left the back side with, at the bottom, the "squadro a ombra" by Institute and Museum of the History of Science (IMSS), Florence (IMSS, 2004). In:

http/brunelleschi.imss.fi.it/explore/astrolabe/index.html.

understand and solve the problems that arose in astronomy. The most widespread instrument was certainly the armillary sphere, known as "spherical astrolabe", a three-dimensional model of the celestial sphere, invented by Eratosthenes in $255 \mathrm{BC}$, formed by rings, or armlets, of metal each of which is one of the reference circles (celestial equator, ecliptic, horizon, hourly circles, tropics, etc.). In the center there is a sphere that represents the Earth; in fact, the mechanism was used to show the movement of the stars around the Earth. There were several types and sizes and some, equipped with appropriate targets and aims, also served as an instrument of observation and measurement, but given their spherical shape they were rather cumbersome. Later it was transformed into a representation of the celestial sphere destined to respond to more complex questions; his golden age began when, in the flat or planispherical version, he could easily solve problems related to sunsets and issues related to the horizon in a given place (Figure 10).

The first information on the development of the astrolabe dates back to 150 BC. In Europe the astrolabe became an indispensable tool for astronomers, astrologers and land surveyors until the end of the seventeenth century when it was replaced by more precise instruments. In the Arab world its use lasted until the 19th century.

From the III century BC until today the term astrolabe has been called instruments that are often very different from each other. They range from the great armillary instruments used to detect the positions of the stars, such as the armillary astrolabe by Claudius Ptolemy (2nd century AD), to modest or small instruments used in astronomical calculations or in navy: the plane astrolabe, the universal astrolabe, the universal astrolabe Rojas, the nautical astrolabe (IMSS, 2004) (Figure 11).

The plane astrolabe is the most important and versatile instrument created in 


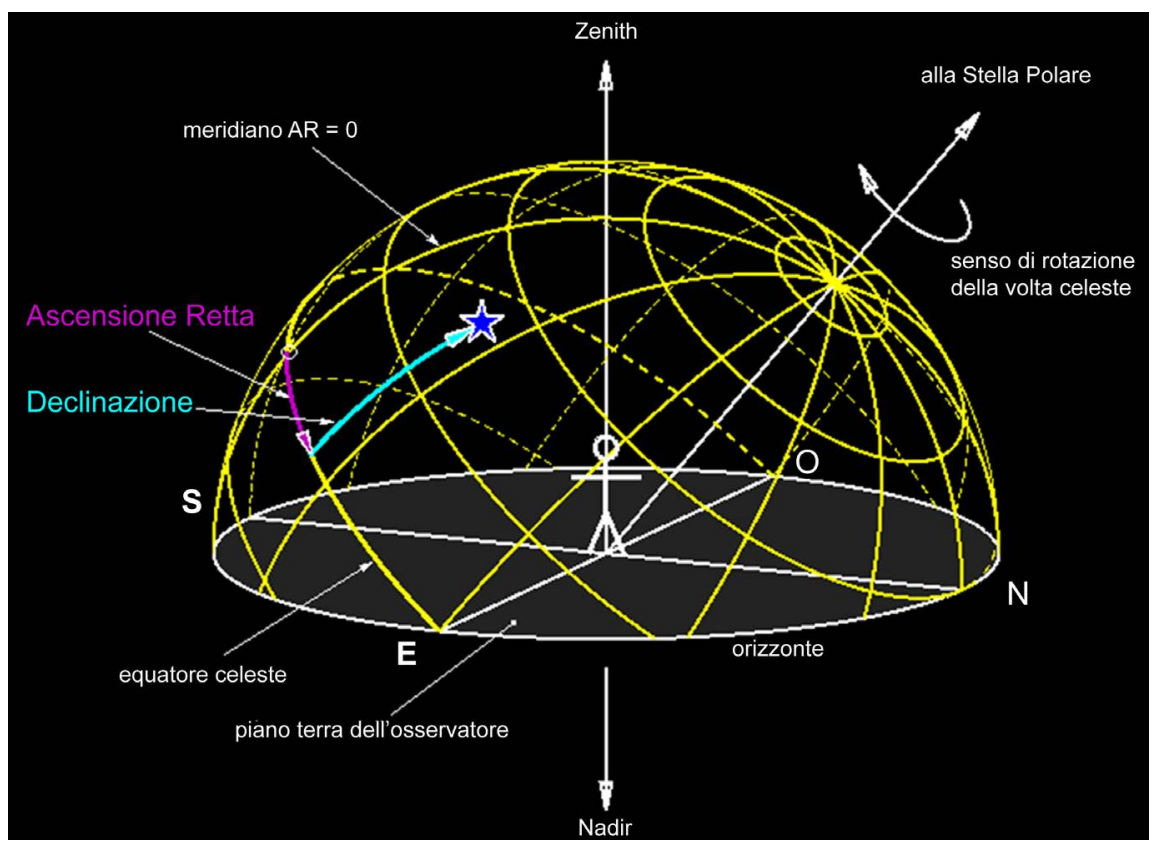

Figure 10. How a star is located by means of the celestial coordinates.

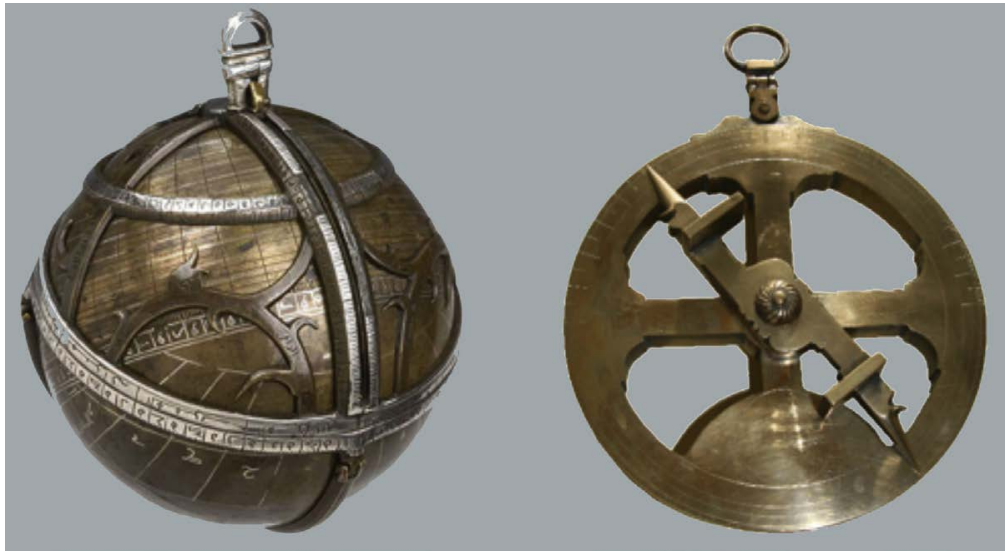

Figure 11. On the left: the spherical astrolabe; on the right: the nautical astrolabe.

antiquity to perform analogical otherwise long and complex astronomical calculations. Some attribute the invention to Hipparchus of Nicaea (II century BC), who knew the stereographic projection used to make it. However, in the "Commentary against the phenomena of Aratus and Eudoxus", Hipparchus used the projection to construct an anaphoric clock which, although similar, is not a plane astrolabe. Three centuries later, Ptolemy exposed the stereographic projection in the "Planisphere", where he hinted at ahoroscopic instrument equipped with a net: perhaps a true plane astrolabe. We can say that the instrument was known to Theone of Alexandria (4th century AD) who has dedicated to it a treatise handed down by Giovanni Filopono (VI century AD) and by Severo Sêbôkht (VII century AD). From Alexandria in Egypt, the instrument passed into Byzantium and into Islam. Here it was developed in all possible variants: from the wooden object used by the astronomer of the mosque to fix the direction of 
Mecca and the hours of prayer through the height of the Sun and the stars, to the instruments of refined workmanship, in silver and precious gems, destined for the princely collections. The Islamic dominion in Spain and in Sicily, as well as the numerous translations of Arabic texts made in the twelfth century-including that of the treaty on the "plane astrolabe" by the astronomer jewish origin Mâshâllâh (mc 815)—, spread in Europe, already from X century, the knowledge of the instrument, which met an undisputed fortune. Only in the seventeenth century, with the construction of accurate mechanical clocks and the elaboration of new methods of calculation, the instrument became obsolete, but without thereby falling into oblivion, in fact even today celluloid astrolabes are sold that allow beginners to identify stars and constellations (Trento, 1989). As previously mentioned, on the tympanum of the plane astrolabe, the grid of the altazimuth coordinates of the observer is found in polar stereographic projection. Since the distance between the zenith and the celestial pole depends on the geographical latitude, the entire grid must be redrawn as soon as you move a few degrees north or south. A single plane astrolabe must therefore be equipped with as many eardrums as there are places of different latitude in which it's to use. This inconvenience was eliminated in Toledo by introducing two new types of astrolabe based on an equinoctial rather than polar stereographic projection. Taken as a point and projection plane, respectively, an equinox and the plane of the solstice, the celestial equator and the horizon of the place are transformed into diameters of the instrument. The celestial poles, as well as the zenith and the nadir, constitute two pairs of diametrically opposite points lying on the circular edge of the instrument. When the observer moves, instead of changing the tympanum, he resets the distance between the zenith and the celestial pole by rotating the network with respect to the mother. The first type of universal astrolabe, due to 'Alî ibn Khalaf al-Shakkâz (11th century), remained almost unknown and was reinvented in Aleppo by Ibn al-Sarrâj (14th century). The mother bears the altazimuth coordinates of the place, while the net contains in one half the grid of the equatorial coordinates, in the other half some indicators of stars. For convenience of calculation the ecliptic is rotated $90^{\circ}$ around the celestial axis and appears in the form of two graduated arches. The second type of universal astrolabe, called "açafeha" or "saphaeaArzachelis" (=plate of al-Zarqâlî), due precisely to the astronomer AbûIbrâhîm ibn Yahyâ al-Zarqâlî (c.1029-c.1087), had more luck. Known in Cairo and Damascus, it was resumed in Europe by Reiner Gemma Frisius (1508-1555) in the "De astrolabiocatholico" (1556). The mother carries the grid of equatorial coordinates and, as a diameter inclined with respect to the celestial equator, the ecliptic. The network is replaced by a graduated alidada with a graduated perpendicular cursor. Universal astrolabes are impractical to use. So, if on the one hand there are only rare remains, on the other the various types testify to the continuous effort of their builders to emulate with them the advantages of the "plane astrolabe". When he wrote the Analemma, Ptolemy also exposed the constructive principle of a different projection from stereography.

The Islamic astronomer Abû al-Raihân al-Bîrûnî (973-c.1050) reworked that 
projection and called it "cylindrical". Taken as a projection plane that of the "colurosolstiziale", each point of the celestial sphere is lowered orthogonally, ie making it slide along its perpendicular to the projection plane. It is not by chance that the "cylindrical" projection of al-Bîrûnî is known today as equinoctial orthographic projection. In the Europe of the second half of the fifteenth century the new projection was used, instead of the equinoctial stereographic projection, to build the mother of several universal astrolabes. As a result of the projection, the parallels and celestial meridians become, respectively, parallel to the celestial equator and elliptic arcs passing through the celestial poles. The ecliptic appears as an oblique segment that intersects the celestial equator in the equinox, located exactly at the center of the mother. In the sixth book of his "Commentarium de astrolabium" (1550), Juan de Rojas y Sarmiento (16th century) provides the first accurate description of a new instrument known as the Rojas universal astrolabe (Figure 12).

As in the al-Zarqâlî universal astrolabe, also in this the net is replaced by a graduated alidade with a graduated perpendicular cursor. The Treaty of Rojas spread throughout Europe the orthographic or cylindrical projection of the earth sphere. The volume was illustrated with sixty-three refined diagrams on the construction and use of the astrolabe described in the text. The work also included material for the construction of solar clocks, shadow squares, and among other things included notes of practical geometry. This projection was used with considerable success in the realization of a new type of astrolabe and represented a great advantage over the previous ones, because it is valid for any latitude. However, as happens in periods of intense innovation and scientific development, Rojas was not the first to investigate in this field and to use the orthographic projection, which was in fact already present in the models of Hans Dorn in Vienna and in those of his master. The nautical astrolabe has little affinity with the plane astrolabe; it shares, next to the overall shape, only the mother with the graduated edge, the support ring and the alidade. The nautical astrolabe was not in fact used in astronomical calculation, but to measure the zenith distance of the Sun or of a star, in particular of the star Polaris, at the moment of transit into the meridian. Knowing the declination, that is the distance from the
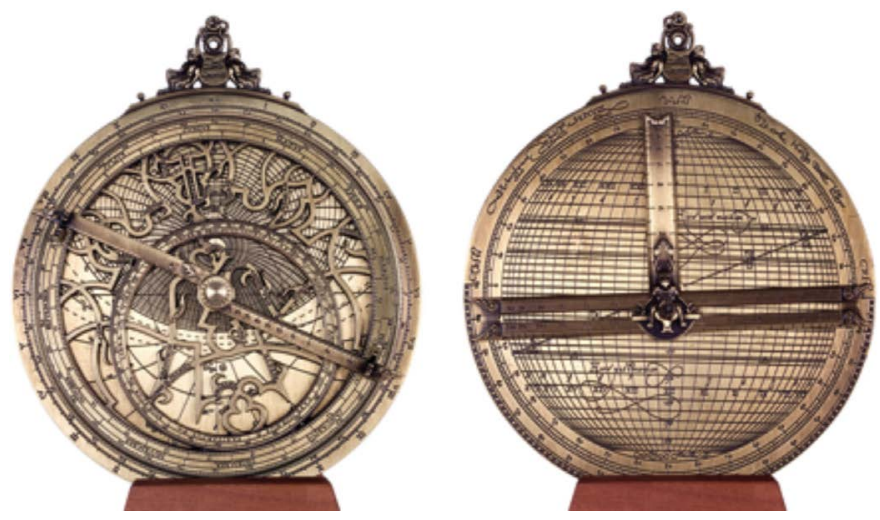

Figure 12. The Rojas astrolabe. 
celestial equator of the star observed one on that given day, the navigator was able to calculate, by sum or subtraction, the latitude touched by the ship. To facilitate observation in adverse weather conditions, the mother of the nautical astrolabe, made of bronze, was thick, heavy and widely perforated. The weight kept the instrument perpendicular to the ground despite the pitch and roll of the ship; the tunnel instead avoided that, in the presence of strong wind, the instrument would sail and oscillate in the observer's hands, preventing it from steadily aiming the chosen star. First present on almost all the great vessels (especially Spanish) from the fifteenth to the seventeenth century, the nautical astrolabe gave way to more manageable and precise instruments up to the modern nautical sextant.

\section{The Functions of the Astrolabe}

The astrolabe provides measurements related to different fields of investigation: astronomy, chronometry, orientation, length-height-depth measurements, astrology.

\subsection{Astronomy: Search for the Position of Celestial Bodies}

1) Almucantarat of the sun and the stars. The celestial vault can be divided into concentric circles (almucantarat) which, starting from the horizon and proceeding towards the zenith, become narrower. On the astrolabe these almucantarat are flattened and eccentric due to the stereographic projection (Figure 13 \& Figure 14).

From the oblique horizon to the zenith there are 90 degrees and to each of them corresponds an almucantarat. The sun, in its apparent daytime motion, intersects the almucantarat (in Arabic: curved lines) according to its degree of inclination. This degree is easily identifiable with the use of alidada and is simply reported on the corresponding almucantarat of the disk. In the stereographic

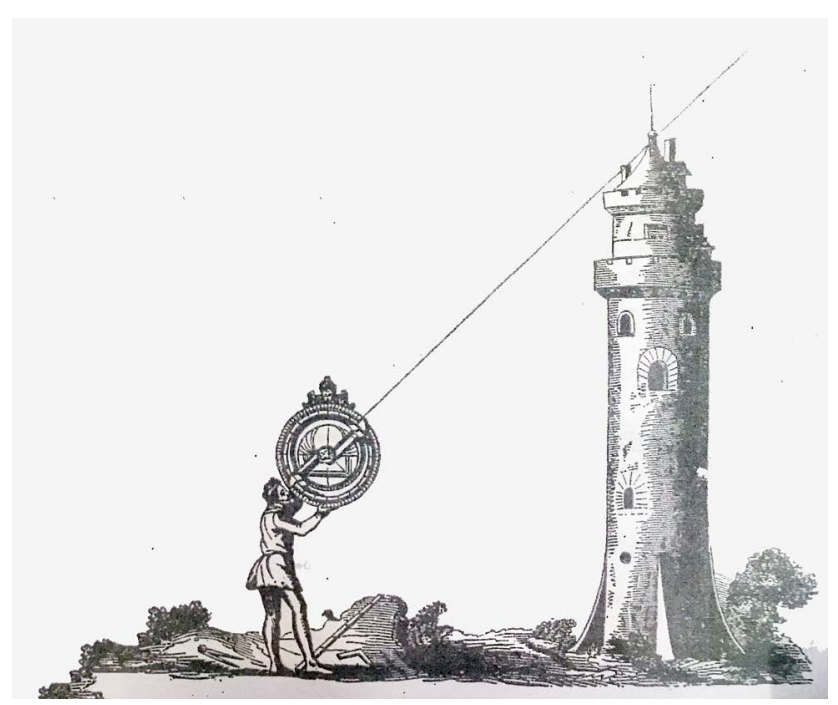

Figure13. Measurement of the position of the stars with the astrolabe. 


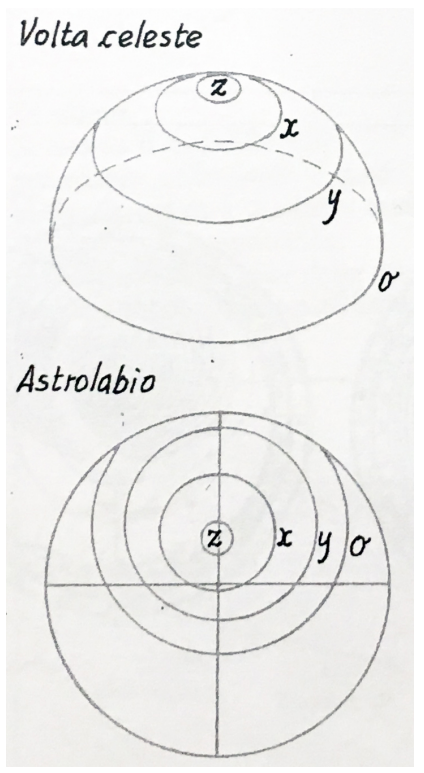

Figure 14. Almucantarat of the celestial vault in the stereographic representation of the astrolabe. $\mathrm{z}=$ zenit; $\mathrm{x}, \mathrm{y}=$ almucantarat; $\mathrm{o}=$ horizon.

representation of the disk, the sun touches the height circle measured in degrees by the alidade.

2) Aracnea position at the moment of observation. At morning: in the geocentric hypothesis, the Earth sees the Sun moving in its annual cycle along the zodiacal circle. In the aracnea grid the path of the sun is represented, according to the epiciclic theory, on an eccentric circle with respect to the center of the astrolabe. So the sun is subject to two operations of calculation: the first concerns the knowledge of its position in the zodiac, i.e. the zodiacal degree on the day of observation, the second concerns the height of the sun at the time of measurement, i.e. the almucatarat (Figure 15).

Placing the zodiacal degree of the day of observation, namely the sun on its almucantarat, aracnea is placed in the searched position. To find the zodiacal degree we use the "trust line" of the alidada. This line, if passed for a given day of the perpetual calendar, indicates the corresponding zodiacal degree (tip of the alidada). Based on height of the sun is the corresponding almucantarat. On the latter, by rotating the aracnea in an appropriate manner, the zodiacal degree is brought, which indicates the position of the sun on the desired day. Obviously the sun, in its apparent diurnal path, passes twice the same almucantarat: before it goes up into the sky (from the east) and after turn (towards the west) at sunset. For this reason it is advisable to know if the height of the sun is relative to the morning or the afternoon, that is before or after 12 o'clock. At night: once a star has been identified the almucantarat is obtained by direct observation before or after midnight. The tip of the aracnea that marks the star is brought to the almucantarat measured with the alidade. Now the aracnea is arranged in the order of the starry vault: each star is in its almucantarat. The problem is analogousthat it for the search for the almucantarat of the stars. About the alidada goals: there 


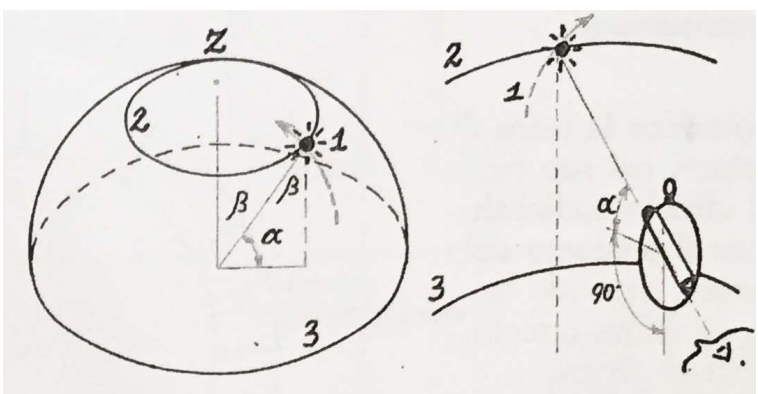

Figure 15. Use of the astrolabe to measure the angle of height of the Sun: $\mathrm{z}=$ zenith; $1=$ apparent motion of the Sun; 2 = almucantarat; 3 = horizon; $a=$ height angle.

are 4 holes, two large and two small. For the observation of the stars we use the largestholes, suitable for detecting the angle of inclination of small light sources; instead the size and brightness of the Sun require the use of smaller holes to obtain accurate measurements.

\subsection{Chronometry or Time Measurement}

1) Equinoctial or astronomical time. Once obtained the zodiacal degree from the calendar it is placed on the almucantarat of the sun, or of a star previously calculated, with the alidade; the ruler, superimposed on the point found, indicates the time of observation on the edge of the astrolabe.

2) Hour unequal. At night: once the aracnea has been regulated on a known star, the zodiacal degree corresponding to the day of the observation touches one of the unequal hour lines below the oblique horizon of the disc. Each line indicates the unequal time: the reading is therefore immediate. At morning: The same procedure is carried out using the opposite zodiacal degree (nadir) to that of the measurement day. The nadir touches an arc of the unequal hours corresponding to the moment of observation.

3) Sunrise and solar sunset. In this operation the oblique horizon line is used: the zodiacal degree of the day established on the quoted line is set (almucantarat 0 ). The ruler, intersecting this almucantarat, will indicate the time of sunrise (left) and sunset (right). The requested number appears on the edge of the astrolabe. With these values you can get the "solar arc", which is the brightness period of each day.

4) Rising and sunset of a star. The same procedure is applied with reference to a known star of the aracnea. Therefore, the zodiacal degree of the solar annual position of the epicycle is not to be calculated.

5) Aurora and twilight. The procedure is identical to that for the determination of sunrise and sunset, except that the line of the aurora and twilight is used instead of the oblique horizon. In practice, the 18th almucantarat is used below the oblique horizon.

\subsection{Orientation}

All the problems of orientation are related to the identification of a path that 
joins two points: one in which we are and the one we must reach. In the absence of other references it is necessary to know the geographic coordinates that, conventionally, are expressed in parallels (latitude) and meridians (longitude).

a) Latitude. It is measured in degrees and expresses the distance of a line, parallel to the equator, from the equator itself. There are two measurement possibilities: the first is nocturnal and is referred to as the polar star; the second is diurnal, but is related to four particular days of the year (equinoxes and solstices). At night: the polar star describes during the 24 hours a tiny circle around the celestial pole. During the winter nights it is possible to observe the polar star at a certain time and repeat the observation exactly 12 hours later. The points detected are diametrically opposed and, in the intermediate position, it is easy to identify the celestial body. Depending on the latitude, in order to observe the polar star, it is necessary to raise the eye by a certain angle to the horizon (angle 0 degrees of inclination): while at the north pole the polar star is exactly at the zenith (angle 90 degrees of inclination), at the equator it appears only on the horizon. With the astrolabe, by identifying the angular height of the polar star, the latitude can be measured with the use of the alidade and the tracing of the grades engraved on the back of the instrument. During the day: the measurement of the latitude is made complex by the inclination of the ecliptic $\left(23^{\circ} 30^{\prime}\right)$ : the shadow of a gnomon measured at midday varies according to the time of year (longer in winter and shorter in the summertime). Only in two days of the period of terrestrial revolution does the plane of the ecliptic intersect the equatorial plane (equinoxes) and it is possible to derive the angle of inclination of the parallel in which the observer is located, i.e. the latitude. In the days of solstice, to obtain the latitude, it will be sufficient to subtract (in summer) and add (in winter) the degrees of inclination of the ecliptic plane $\left(23^{\circ} 30^{\prime}\right)$ at the angle measured with the gnomon. All these operations are carried out immediately using the astrolabe.

b) Longitude: is linked to the measurement of time, since on the same meridian it is the same time at the same time. The meridians are 360 , one for each degree of the equatorial circle and the Earth performs an entire rotation every 24 hours: at each hour there are 15 meridians calculated from a main meridian (meridian zero). For the calculation of longitudes there are two orders of problems: the first about chronometry linked to the technology of the various epochs, the second about convention, to establish a zero meridian valid for all nations.

The astrolabe is also important in nautical orientation as evidenced by documents that tell of Magellano that in his journey of circumnavigation of the globe as well as other instruments had with him a large wooden astrolabe (reproduced in the Naval Museum of Pegli) and six metal astrolabes. Before the great geographical discoveries, longitude was calculated as a chronological difference between the observations of the same phenomenon (for example an eclipse) from two different places. Another system, presumably used by Colombo, used special boards, called ephemerides, to determine longitude by the distance of the moon from known planets. But even this method was liable to errors because of the difficulty of assessing the diameter of the celestial bodies with the naked eye. The 
idea of determining longitude with the transport of time is attributed to Fernando, son of Christopher Columbus, who in 1524 exposed to Philip II of Spain the need for new chronometric instruments. For the choice of the zero meridian, only in 1844 was accepted unanimously the meridian of Greenwich (given the English naval dominance).

\subsection{Body Measurement: Height, Length, Depth}

This function of the astrolabe allowed the survey of the architectures. These measures are provided by the "squadro a ombra", placed on the back of the astrolabe, through the movement of the alidade. In the following formulas the abbreviations are used: $M=$ maximum index value; $I=$ index; $A c=$ height of the body; Ao = height of the observer; $A^{\prime} \mathrm{o}=$ projection of the height of the observer; $\mathrm{d}=$ distance of the observer from the base of the body; Oc = shadow of the body; $\mathrm{Am}=$ height of the metric rod.

1) Buildings with an accessible base

a) Measurement of height without the use of shadows. This problem is solved by placing the alidade at $45^{\circ}$ : the observer moves away from the body until he succeeds in drawing from the holes of the alidade a point (A) representative of the height of the object to be measured. The distance from where is the observer to the building, added to its height, returns the measure of the height sought. In the application formula, this translates as follows: $A=d+$ Ao. For example: a man $1.70 \mathrm{~m}$ tall who measures the height of a building taking the alidade at $45^{\circ}$ and reaching its highest point, if it runs $22 \mathrm{~m}$ to the base of the building, the building will be 23.70 high $\mathrm{m}$.

b) Measurement of heights with the shadow produced by the sun or the moon with inclination greater than $45^{\circ}$. When the shadows are shorter than the heights to be measured, the formula is applied: $\mathrm{A}=(\mathrm{Oc} \times \mathrm{M}) / \mathrm{I}$.

The index I is measured on the side of the "squadro a ombra", holding the alidade so as to be able to reach a point of unknown height. For example: the shadow of a house is $4 \mathrm{~m}$, the index of the astrolabe measures on the horizontal side 8 . The height of the building will be 5 meters. $(4 \times 10) / 8=5$ (Figure 16).

2) Buildings with inaccessible base

The condition for making the measurements is that the buildings are erected on level ground and, above all, that the observer can approach their base. Otherwise it is necessary to resort to other proceedings. The first concerns the calculation of a length, for example a river bed or a moat trough, by using a metric rod erected near the observer. In practice, since the problem is the opposite of the previous ones, it is sufficient to reverse the terms: $d=(A m \times M) / I$ (Figure 17 \& Figure 18).

To calculate the height of a building placed on a rise it is necessary to perform this procedure twice: the first at the point of maximum height, the second on the inaccessible base. The required value is given by the subtraction of the two measures obtained. 


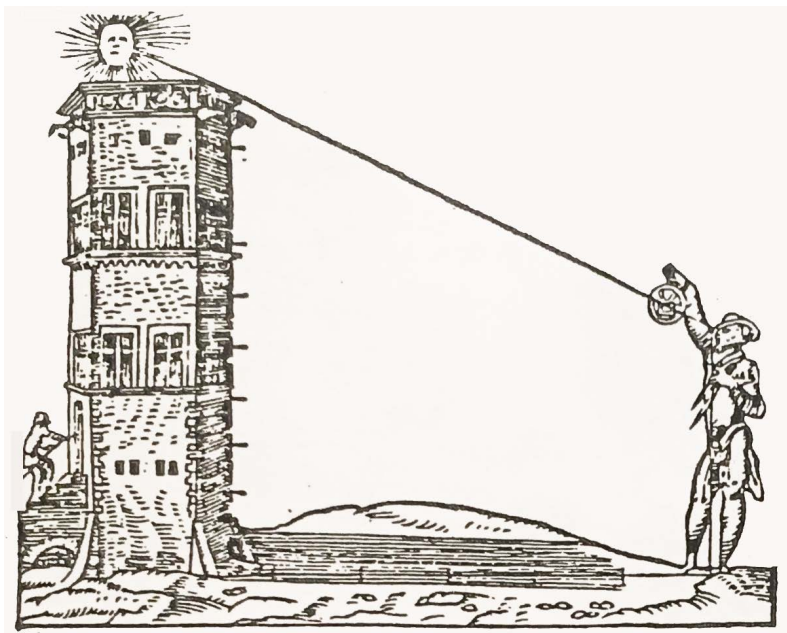

Figure 16. 3. Measurement of heights with the shadow produced by the sun or the moon with inclination less than $45^{\circ}$. When the shadows are greater than the heights to be measured, the formula is applied: $\mathrm{A}=(\mathrm{Oc} \times \mathrm{I}) / \mathrm{M}$. In this case the measurement of the index I isobtained on the vertical side of the shadow diagram.

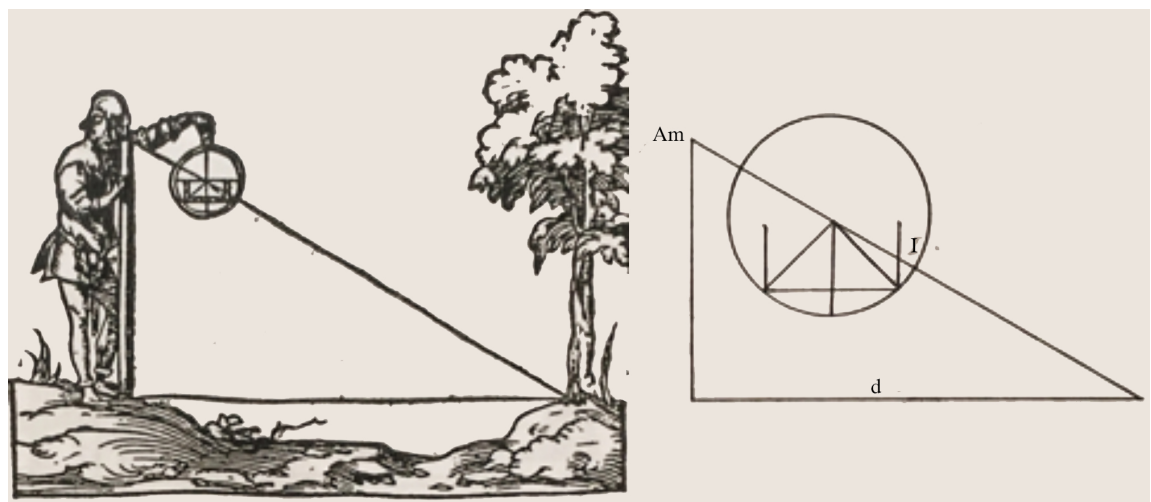

Figure 17. For example: with a $1.5 \mathrm{~m}$ high metric rod and the alidade arranged as in the figure, we want to measure the length of a road. The index marks 5 then the road will be 3 m long: $(1.5 \times 10) / 5=3$.

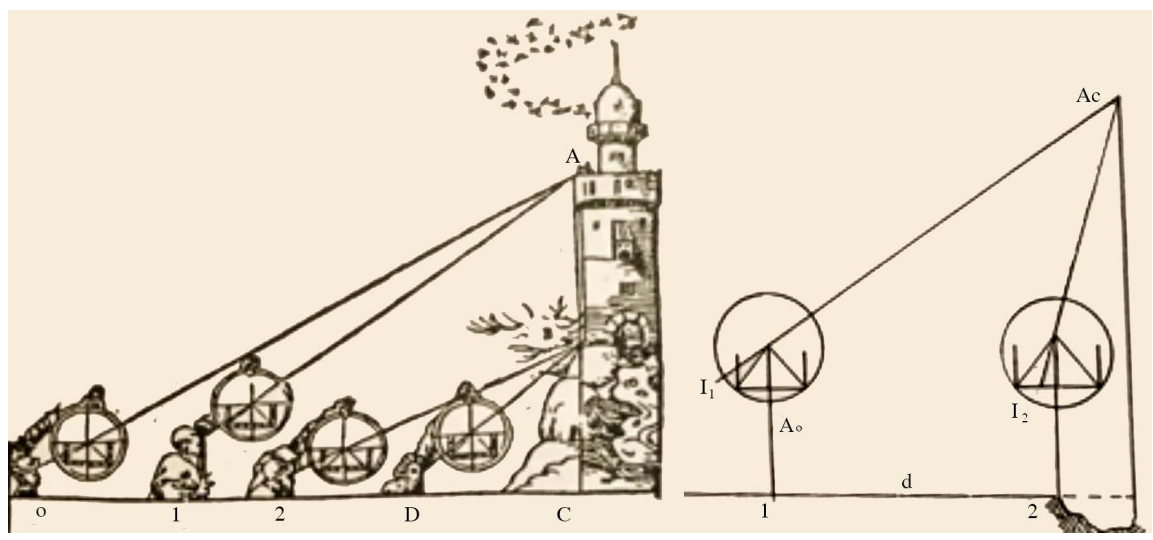

Figure 18. To calculate the height of a body whose base is inaccessible it is sufficient to find two indices $\left(I_{1}\right.$ and $\left.I_{2}\right)$ in two points whose distance, approaching towards the body of unknown height, is measurable. The formula applied is: $A c=\left(M / I_{1}-M / I_{2}\right)+A o+d_{1}$ $-\mathrm{d}_{2}$. 


\section{3) Depth of a well}

The data available are the diameter of the well and the index of the shadow squared (Figure 19). The diameter is multiplied by $M$ (in the metric case equal to 10). This product is divided by the index (I). The result is the depth of the well $(P) . P=(\mathrm{F} \times 10) / \mathrm{I}$.

\subsection{Astrology}

The main astrological functions of the astrolabe are linked to the "celestial theme", this is a representative scheme of the characteristics of the individual, based on the principle that at the time of birth the arrangement of celestial bodies is unique and characteristic for each individual, as happens for the Fingerprints. This scheme, once interpreted, outlines a person's biographical and psychological portrait and is therefore called "astral picture" (Trento, 1989). Furthermore according to an ancient belief the influence of the celestial bodies affect the fate of the hours of the day, divided into periods of different duration depending on the time of year (unequal hours). For this reason the sequence of the days of the week referred to the planets has an astrological meaning starting from the Moon-Monday, Mars-Tuesday, up to Saturn-Saturday and to the Sun-Sunday. The "table of influential planets" was present in all the sixteenth-century treatises on the astrolabe. In astrology the planets have a particular symbology linked to mythology, but also to very ancient observations on nature. Thanks to the astrolabe it is possible to perform astrological operations such as the search for the ascendant, that of the zodiacal sign in the twelve houses of the sky or that of the influential planet on the unequal hour.

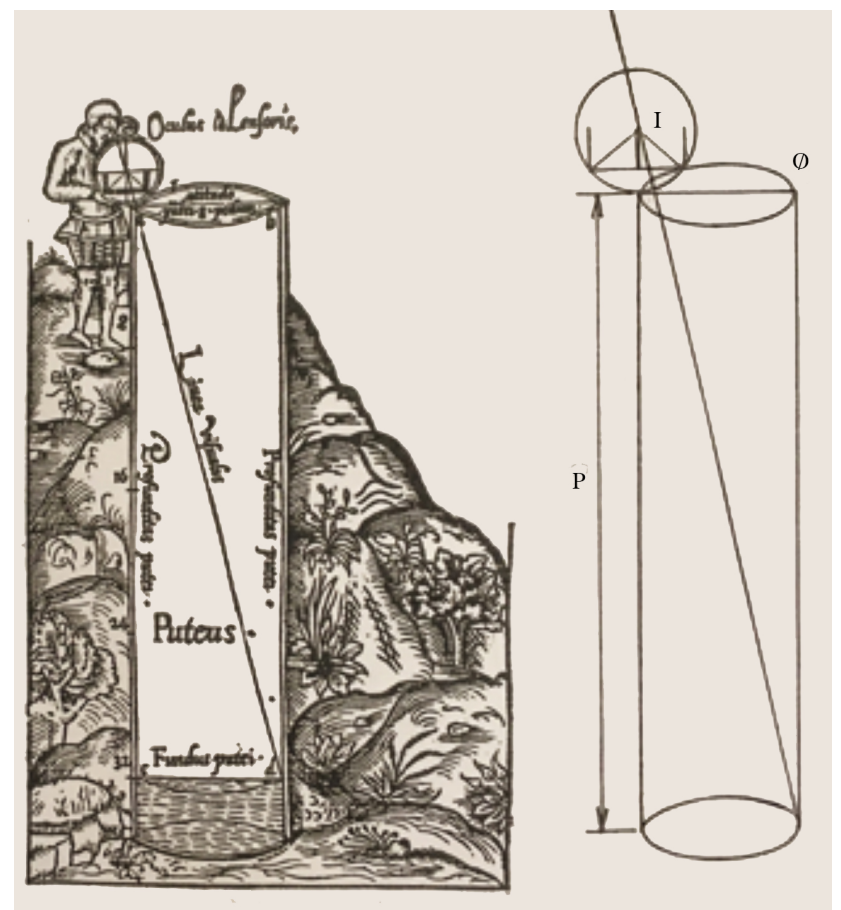

Figure 19. The measurement of the depth of a well through the astrolabe. 


\section{Conclusion}

The astrolabe is a complete and refined instrument that has its roots in past centuries, just think that the Greeks used it already two centuries before the birth of Christ.

As we said, it is based on stereographic projection, so we can define it as a practical application of the principles of projective geometry.

Observing him astonishing to notice that the knowledge of this discipline was so high, even many centuries ago, so as to be able to conceive such a sophisticated mechanism but also simple to use. Such a precise instrument was fundamental both for astronomers, who used it for their observations, and for merchants and travelers, who had to know the time (day or night) and know where they were traveling.

Particularly it has played a fundamental role in sea voyages and, even if today it has been replaced by the modern sextant, it remains a scientific instrument of great value and charm, so much so that we can think of its unlimited use over time.

Another important use was in the survey, as it allowed to measure heights and depths even of inaccessible objects. Measuring the height of a tower or the depth of a well would have been impossible before without the aid of this tool.

So the astrolabe is certainly one of the most guessed mechanical instruments in history.

\section{Conflicts of Interest}

The author declares no conflicts of interest regarding the publication of this paper.

\section{References}

Aterini, B. (2019). The Astrolabe: A Mechanism for Reading the Stars. In M. Ceccarelli, \& B. Zhang (Eds.), Explorations in the History and Heritage of Machines and Mechanisms (pp. 227-242). Switzerland: Springer Nature.

Fantoni, G. (1988). Orologi solari. Trattato completo di gnomonica, Ed. Roma: Technimedia.

IMSS (2004). Istituto e Museo di Storia della Scienza (IMSS), Firenze. http://brunelleschi.imss.fi.it/esplora/astrolabio/indice.html

Trento, P. (1989). Astrolabio. Storia, funzioni, costruzione. Biblioteca del Vascello. 